\title{
Note on the Equivariant $K$-Theory Spectrum
}

\author{
To the memory of Professor Masahisa Adachi
}

By

\author{
Kazuhisa SHIMAKAwA*
}

\section{Introduction}

Let $G$ be a finite group. For any finite $G$-CIV complex $X$, denote by $K O_{G}(X)$ the equivariant real $K$-theory of $X$ and $\operatorname{Sph}_{G}(X)$ the group of stable equivalence classes of spherical $G$-fibrations over $X$.

In [9] and [10], we showed that there are connective $G$-spectra $k O_{G}$ and $k F_{G}$ representing the theories $K O_{G}(-)$ and $\operatorname{Sph}_{G}(-)$ respectively. and a map of $G$-spectra $k O_{G} \rightarrow k F_{G}$ inducing the equivariant $J$-homomorphism

$$
J_{G}: K O_{G}(X) \longrightarrow \operatorname{Sph}_{G}(X) .
$$

(Cf. Theorem 4.3 of [10].) It follows, by [8], that $K O_{G}(-)$ and $\operatorname{Sph}_{G}(-)$ are equipped with transfers for $G$-equivariant bundles which are compatible with $J_{G}$.

In this note, we will show that $k O_{G}$ is, in fact, a $(-1)$-connected cover of the $G$-spectrum representing the periodic $\mathrm{KO}_{G}$-theory; that is, the equivariant infinite loop structures defined by $k O_{G}$ and by the Bott periodicity are equivalent to each other. Thus, the transfer associated with $k O_{G}$ coincides with the transfer coming from the Bott periodicity.

We also apply our results to deduce the equivariant Adams conjecture for arbitrary finite groups from one and two dimensional cases proved by HauschildWaner [4]. (Compare [2] and [7].)

\section{$\S 1$. Construction of the Periodic $K O_{G}$-Theory Spectrum}

This section is devoted to the proof of the following theorem. (For the definition of $G$-spectrum, see [6].)

Theorem 1. There are a $G$-spectrum $K O_{G}$ representing the periodic $K O_{G^{-}}$ theory and a map of $G$-spectra $l_{G}: k O_{G} \rightarrow K O_{G}$, defined in the stable category, inducing an equivalence between $k O_{G}$ and the $(-1)$-connected cover of $K \mathrm{KO}_{G}$.

Communicated by Y. Ihara, June 19, 1992.

1991 Mathematics Subject Classifications : 19L47, 55R91

* Department of Mathematics, Faculty of Science, Okayama University, Okayama 700, Japan 
We begin by recalling from [9] and [11] the construction of $k O_{G}$. For each $n \geqq 0$, let $O_{n}$ be the real orthogonal group regarded as a $G$-category with unique object and with trivial $G$-action. Then the union $\amalg_{n \geq 0} O_{n}$ can be regarded as a bipermutative $G$-category with respect to Whitney sum and tensor product operations. By Theorem A of [11], we obtain an $E_{\infty}$ ring $G$-spectrum

$$
k O_{G}=E_{G}\left(\operatorname{I}_{n \geq 0} O_{n}\right)
$$

indexed on $A=\left\{V^{n} ; n \geqq 0\right\}$, where $V$ is a real Spin $G$-module of dimension $8 n$ such that every irreducible representation of $G$ can be embedded in $V$.

For brevity, write $k O_{G}=\left\{E_{n} ; n \geqq 0\right\}$, so that $E_{0}=\Omega^{\infty} k O_{G}$. We showed in $[9, \S 3]$ that $E_{0}$ is $G$-equivalent to the homology theoretic group completion of the $G$-monoid $\amalg_{n \geq 0} B O_{n}(G)$ where $B O_{n}(G)$ is a classifying space for $n$-dimensional real $G$-vector bundles. Thus we have

$$
K O_{G}(X)=\left[X^{+}, E_{0}\right]^{G}=\left[\Sigma^{\infty} X^{+}, k O_{G}\right]^{G}
$$

for every finite $G-\mathrm{CW}$ complex $Y$.

As in [11], let $\mathcal{C}$ be an $E_{\infty} G$-operad with $\mathcal{C}_{j}=\left|\operatorname{Cat}\left(E G, E \Sigma_{j}\right)\right|$ where $E G$ denotes the translation category of $G$, that is, ob $E G=G$ and mor $E G=G \times G$ (and similarly for $E \Sigma_{j}$ ). Then $k O_{G}$ comes equipped with an $E_{\infty}$ ring structure defined by external $\mathcal{C}$-action

$$
\xi_{j}: \mathcal{C}_{J}^{+} \wedge E_{n_{1}} \wedge \cdots \wedge E_{n_{j}} \rightarrow E_{n_{1}+\cdots+n_{j}} ; \quad j \geqq 0, n_{1}, \cdots, n_{j} \geqq 0 .
$$

Let $\iota_{2}$ be the element of $\mathcal{C}_{2}$ represented by the constant $E G \rightarrow E \Sigma_{2}$ with value 1 , and let $\mu: E_{0} \wedge E_{0} \rightarrow E_{0}$ be a multiplication defined by

$$
\mu(x \wedge y)=\xi_{2}\left(\iota_{2} \wedge x \wedge y\right) .
$$

One easily verifies that $\mu$ is strictly associative and homotopy commutative.

Let $S V$ be the onepoint compactification of $V$ and let $b: S V \rightarrow E_{0}$ be a $G$ map representing the Bott class in $\widetilde{K O}_{G}(S V)$. We define a $G$-prespectrum $D=$ $\left\{D_{n} ; n \geqq 0\right\}$ as follows. For each $n \geqq 0$, put $D_{n}=E_{0}$ and define its structure map $\delta: D_{n} \wedge S V \rightarrow D_{n+1}$ to be the composite

$$
D_{n} \wedge S V \stackrel{1 \wedge b}{\longrightarrow} E_{0} \wedge E_{0} \stackrel{\mu}{\longrightarrow} E_{0}=D_{n+1} .
$$

By the Bott periodicity theorem, the homomorphism $\widetilde{K O}_{G}(X) \rightarrow \widetilde{K O}_{G}(X \wedge S V)$ induced by the adjoint $E_{0} \rightarrow \Omega^{V} E_{0}$ to $\delta$ is an isomorphism (cf. [1]). Hence $D$ is an $\Omega$-G-prespectrum and represents the periodic $K O_{G}$-theory.

Let $K O_{G}$ denote the $G$-spectrum associated to $D$. We construct a map of $G$-spectra $k O_{G} \rightarrow K O_{G}$ by using the equivariant version of the up and across theorem. (Compare the proof of theorem 2.4 in [11].)

Let $A \oplus \mathcal{A}$ denote the indexing set $\left\{V^{m} \oplus V^{n} ; m, n \geqq 0\right\}$ in the universe 
$V^{\infty} \oplus V^{\infty}$, and define a $G$-prespectrum $X=\left\{X_{m, n}\right\}$ indexed on $A \oplus \mathcal{A}$ as follows. For $m, n \geqq 0$, put

$$
X_{m, n}=E_{n}
$$

and define $G$-maps $\chi^{\prime}: X_{m, n} \wedge S V \rightarrow X_{m+1, n}, \chi^{\prime \prime}: X_{m, n} \wedge S V \rightarrow X_{m, n+1}$ by

$$
\chi^{\prime}(x \wedge v)=\xi_{2}\left(\iota_{2} \wedge x \wedge b(v)\right), \quad \chi^{\prime \prime}(x \wedge v)=\varepsilon(x \wedge v)
$$

where $\varepsilon$ denotes the structure map of $k O_{G}$.

By the property of external $\mathcal{C}$-action (cf. Definition 12 (1.3) of [11]), the following diagram is commutative:

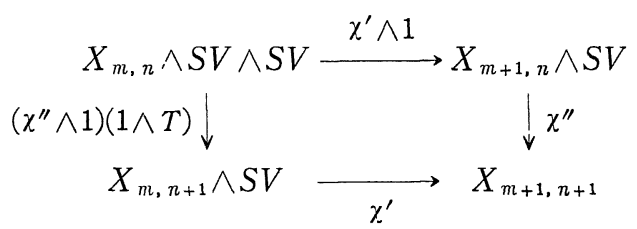

where $T$ denotes the transposition $u \wedge v \mapsto v \wedge u$. It follows that $X_{m, n}$ together with $\chi^{\prime}$ and $\chi^{\prime \prime}$ determine a $G$-prespectrum $X \equiv G \mathscr{L}(\mathcal{A} \oplus \mathcal{A})$.

Let $i^{*}$ and $j^{*}$ be the functors $G \mathscr{P}(A \oplus \mathcal{A}) \rightarrow G \mathscr{P} A$ induced by the embeddings of $V^{\infty}$ into the first and the second factor of $V^{\infty} \oplus V^{\infty}$ respectively, and let $L$ be the functor which takes $G$-prespectra to their associated $G$-spectra. Then $X$ satisfies the following properties:

(1) $L i * X=K O_{G}, L j * X=k O_{G}$.

(2) The natural map $L i^{*} X \rightarrow i^{*} L X$ is a $G$-equivalence.

Moreover, there is a $G$-equivalence $j^{*} L X \cong i^{*} L X$ uniquely determined in the stable category. (See Theorem 1.7 of [6, Chapter II].) Thus we get a map of $G$-spectra $l_{G}: k O_{G} \rightarrow K O_{G}$ defined by the composite

$$
k O_{G}=L j^{*} X \rightarrow j^{*} L X \cong i^{*} L X \cong L i^{*} X=K O_{G} .
$$

It is easily verified that $l_{G}$ induces isomorphisms $\pi_{n}^{H} k O_{G} \cong \pi_{n}^{H} K O_{G}$ for all subgroups $H$ and $n \geqq 0$. Therefore, $k O_{G}$ is equivalent to the $(-1)$-connected cover of $K O_{G}$.

Note. The arguments above remain true if $K O_{G}$ is replaced by $K_{G}$ or $K R_{G}$. Thus we have complex and Real analogs of Theorem 1. Moreover, $l_{G}$ can be taken to be a map of $H_{\infty}$ ring spectra, which we hope to discuss elsewhere.

\section{§. The Equivariant Adams Conjecture}

We now give a proof of the equivariant Adams conjecture formulated by McClure [7]. (For a slightly different formulation, see Fiedorowicz-HauschildMay [2].) 
For any finite $G$-CW complex $X$ and every prime $p$, let $\operatorname{Sph}_{G}^{(p)}(X)$ denote the quotient of $\operatorname{Sph}_{G}(X)_{(p)}$ under the equivalence relation induced by stable $p$ equivalence (cf. $[7, \S 1]$ ). Let $J_{G}^{(p)}$ be the composite

$$
K O_{G}(X)_{(p)} \stackrel{J_{G}}{\longrightarrow} \operatorname{Sph}_{G}(X)_{(p)} \longrightarrow \operatorname{Sph}_{G}^{(p)}(X) .
$$

Theorem 2. Let $X$ be a finite $G-C W$ complex, and $x \Subset K O_{G}(X)$. If $k$ is prime to $p$ and the order of $G$ then $J_{G}^{(p)}\left(\psi^{k} x-x\right)=0$.

We prove this theorem by generalizing the arguments of Hashimoto [3]. (See also Nishida [8] and Kono [5].)

Observe first that it suffices to prove the case where $x$ is the class of an odd dimensional $G$-vector bundle. Let $\xi$ be a $(2 m+1)$-dimensional real $G$-vector bundle over $X$ and $E$ the total space of the associated principal $G-O_{2 m+1}$ bundle. For a closed subgroup $K$ of $O_{2 m+1}$, let $\alpha: R O(K) \rightarrow K O_{G}(E / K)$ be a homomorphism which takes $K$-vector spaces $V$ to the $G$-vector bundles $E \times{ }_{K} V \rightarrow E / K$, and let

$$
\pi_{!}: K O_{G}(E / K) \rightarrow K O_{G}(X), \quad \pi_{!}: \operatorname{Sph}_{G}(E / K) \rightarrow \operatorname{Sph}_{G}(X)
$$

be the transfers, in the sense of [8], for the projection $\pi: E / K \rightarrow X$. Theorem 1 implies that the first $\pi_{\text {: }}$ is identical with the transfer coming from the Bott periodicity.

By the argument of [8, Proposition 3] and the fact that $J_{G}$ is induced by a map of $G$-spectra, we obtain

Proposition 3. The following diagram is commutative

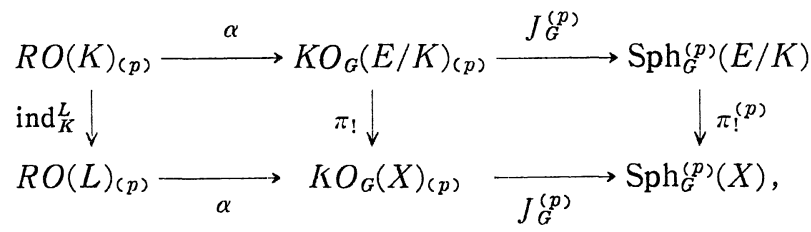

where $L=O_{2 m+1}$ and $\pi_{!}^{(p)}$ is induced from the transfer by passing to quotient.

We are now ready to prove Theorem 2. Put $K=O_{2} \times O_{2 m-1}$ and let $c$ be the identity representation of $L=O_{2 m+1}$, so that $\xi=\alpha(\iota)$. By [3, Proposition 5], there exist a one dimensional representation $\nu$ of $L$ and a two dimensional representation $\mu$ of $K$ such that

$$
\iota=\operatorname{ind}_{K}^{L} \mu+\nu .
$$

Since Theorem 2 is true for one and two dimensional $G$-vector bundles by Hauschild-Waner [4], we have 


$$
\begin{aligned}
J_{G}^{(p)}\left(\psi^{k}-1\right)(\xi) & =J_{G}^{(p)}\left(\phi^{k}-1\right) \alpha(\iota) \\
& =J_{G}^{(p)}\left(\phi^{k}-1\right) \alpha\left(\operatorname{ind}_{K}^{L} \mu\right)+J_{G}^{(p)}\left(\psi^{k}-1\right) \alpha(\nu) \\
& =J_{G}^{(p)}\left(\phi^{k}-1\right) \pi_{!} \alpha(\mu)+J_{G}^{(p)}\left(\psi^{k}-1\right) \alpha(\nu) \\
& =\pi: J_{G}^{(p)}\left(\phi^{k}-1\right) \alpha(\mu)+J_{G}^{(p)}\left(\phi^{k}-1\right) \alpha(\nu) \\
& =0 .
\end{aligned}
$$

This completes the proof of Theorem 2 .

\section{References}

[1] Atiyah, M.F., Bott periodicity and the index of elliptic operators, Quart. J. Math. Oxford Ser. (2), 19 (1968), 113-140.

[2] Fiedorowicz, Z., Hauschild, H. and May, J.P.. Equivariant algebraic $K$-theory, Lecture Notes in Math., 967 (1982), 23-80.

[3] Hashimoto, S., The transfer map in the $K R_{G}$-theory, Osaka J. Math., 18 (1981), 501-507.

[4] Hauschild, H. and Waner, S., The equivariant Dold theorem mod $k$ and the Adams conjecture, Illinois J. Math., 27 (1983), 52-66.

[5] Kono, A., On the order of certain elements of $J(X)$ and the Adams conjecture, Publ. RIMS, Kyoto Univ., 17 (1981), 557-564.

[6] Lewis, G., May, J.P. and Steinberger, M., Equivariant stable homotopy theory, Lecture Notes in Math., 1213 (1986).

[7] McClure, J.E., On the groups $J O_{G} X$. I, Math. Z., 183 (1983), 229-253.

[8] Nishida, G., The transfer homomorphism in equivariant generalized cohomology theories, J. Math. Kyoto Univ., 18 (1978), 435-451.

[9] Shimakawa, K.. Infinite loop $G$-spaces associated to monoidal $G$-graded categories, Publ. RIMS, Kyoto Univ., 25 (1989), 239-262.

[10] -, Mackey systems of monoidal categories and $G$-spectra, K-Theory, 5 (1992), 373-394.

[11] - An $E_{\infty}$ ring $G$-spectrum representing the equivariant algebraic $K$-theory of a bipermutative G-category, Quart. J. Math. Oxford Ser. (2), to appear. 
